\title{
Land Value Taxation: A Spatially Explicit Economic Experiment with Endogenous Institutions
}

\author{
Joshua M. Duke ${ }^{1}$ (1) $\cdot$ TianHang Gao
}

Accepted: 19 October 2021

(C) The Author(s) 2021

\begin{abstract}
An economic experiment with endogenous institutions informs the political economy of land value taxation relative to uniform property taxation in terms of efficiency and sprawl reduction. Heterogeneous type distributions were used so that land value taxation was earnings-rational, relative to uniform property taxation, for 40,60 , and 80 percent of the participants. The model's induced values predict land value taxation leads to less sprawl, more earnings, and more tax revenue than uniform property taxation. Experimental data do not consistently match this prediction, where both tax institutions led to more sprawl and lower earnings than predicted. Results show participants voted for the tax institution that does not maximize their individual earnings in 16.7 percent of rounds. These earnings-irrational choices occurred when the type distributions were 40 and 60 percent in favor of land value taxation. The experiment results nonetheless show the absolute advantage of land value taxation for producing less sprawl, more tax revenue, and more earnings. Moreover, the behavioral evidence suggests that relative advantage of land value taxation in reducing sprawl is greater than predicted by the model. This suggests further inquiry about whether land value taxation promotion activities may best be targeted towards cities using uniform property taxation where economies are vibrant, land uses are already relatively intensive, and greater-than-average population density already exists.
\end{abstract}

Keywords Land use · Property taxation · Public policy

Joshua M. Duke

duke@auburn.edu

TianHang Gao

gaoth@udel.edu

1 Department of Agricultural Economics and Rural Sociology, Auburn University, Auburn, AL, USA

2 Department of Economics, University of Delaware, Newark, DE, USA 


\section{Introduction}

Many researchers believe the land tax (LVT) is superior to and more efficient than uniform property taxation (UPT), in part, because it promotes a distortionless, more-intensive use of land (Pollock \& Shoup, 1977; DiMasi, 1987; Plassmann \& Tideman, 2000; England \& Ravichandran, 2010; Banzhaf \& Lavery, 2010; Chapman et al., 2009; Choi \& Sjoquist, 2015; Gemmell et al., 2019) without regressivity (Bowman \& Bell, 2008; Choi \& Sjoquist, 2015; Plummer, 2010) or with correctible levels of regressivity (England \& Zhao, 2005). An important corollary to the intensity-efficiency advantage is that LVT also prevents "sprawl." Sprawl is a value-laden word, but the meaning to an economist is that land use develops with negative technological externalities. Just as LVT "gets prices right" with respect to the intensity of vertical improvements, LVT also can help get prices right horizontally. LVT's horizontal efficiency arises not from internalizing negative externalities but instead from maximizing the economic potential of land near the central business district (CBD) and reducing incompatible land uses.

Greater-than-anticipated land investment, capital/land ratios, and population density have become metrics for the spatial land-use advantage of LVT. The relevant studies use different models, data, and/or metrics to assess the spatial impacts of LVT, so it is not necessarily clear how to synthesize the results (though Banzhaf \& Lavery, 2010 offer the most comprehensive work). Pollock and Shoup (1977) presented an early approach to the investment-intensity question, arguing that LVT could increase improvements by up to 25 percent. DiMasi (1987) used a general equilibrium model to show that a version of LVT increases improvements per unit of land and population density. Plassmann and Tideman (2000) offered empirical evidence that land tax municipalities had significantly higher levels of construction (measured with building permits). Banzhaf and Lavery (2010) offered strong, empirical evidence that a version of the land tax reduces sprawl because it increases housing units but not housing size. Importantly, Banzhaf and Lavery (2010) argued that perceived inconsistencies in the LVT-sprawl papers (c.f. Choi \& Sjoquist, 2015 vs. Song \& Zenou, 2006) may be because different metrics are being confounded; LVT has an improvement effect, a density effect, and a dwelling-size effect (also see England et al., 2013 for a study of these effects with varying property tax). On balance, the literature suggests that moving from UPT to LVT will reduce sprawl and increase the intensity with which land is used (Brueckner \& Kim, 2003; Choi \& Sjoquist, 2015).

This paper offers a spatial-economic model and experiment of a stylized city, where there is predicted efficiency, tax revenue, and density advantage for LVT relative to UPT. Economic experiments are new to LVT research, and the first experiment paper (Duke \& Gao, 2018) offered a land investment model with endogenous institutional choice treatments, i.e., experiment participants were able to vote for their preferred tax institution while making their land-investment choices. This paper offers a new model with two further innovations. First, the new model is spatially explicit, which provides insight on how tax institutions affect sprawl. Second, the distribution of types varies to examine how the size 
of the majority (or minority) in favor of LVT affects voting patterns; this helps inform a persistent question in LVT research about how many "winners" from LVT are needed to generate support for political change. Although the model parameterization is simplified so that experiment participants can understand it, the model's predicted LVT efficiency advantage corresponds to previous literature and intuition-land-use improvement faces no deadweight losses from taxation and cities will have fewer land-use-coordination diseconomies.

The political economy perspective is informative because prior research suggests that LVT faces a vast array of political objections (c.f. Fischel, 2015, p. 15; Youngman, 2016, p. 193). LVT objections were explored by Bourassa (2009, pp. 195-6) who found ethical objections (such as opposition to taxing unrealized capital gains), objections from policy change "losers," and objections to toodense development. Plummer (2009) investigated perceived inequities from tax incidence. Duke and Gao (2018) found that despite a general tendency to be more efficient than UPT, LVT may trigger over-investment among some types because of positionality. This paper develops an entirely new experiment to remove some drivers of LVT effectiveness identified in Duke and Gao (2018), including positionality and regressivity corrections. The results herein show that experiment participants sometimes vote for the tax that does not maximize their individual earnings ("earningsirrationality") and group patterns can affect institutional choice.

\section{Methods}

This section explains the spatial-economic model that governs the experiment and leads to predictions. The experiment explores how cities grow spatially under different tax plans with a stylized model centered on an existing central business district (CBD). As seen in Fig. 1, undeveloped land is available for building at four unit intervals, labeled "Ring 1" to "Ring 4." For simplicity, experiment participants $j=1, \ldots, 5$ act as developers ${ }^{1}$ who own one of five "roads," which are rays emanating from the CBD. Each road owner can develop land at four locations $i=1, \ldots, 4-$ one for each ring. Thus, there are 20 potential development locations indicated by $(i, j)$ pairs. Undevelopable agricultural land is beyond the fourth ring. Because this model leads to an experiment, the decision problem is simplified to fit participants' comprehension capabilities during a single session. A round consists of four periods, $t$. In each period, the participant decides at which location $i$ to place a single development unit, which is stylized as a "brick." A brick corresponds to land-use intensification or the improvement value in other papers. $B_{i j t}$ denotes the number of bricks

\footnotetext{
${ }^{1}$ Duke and Gao (2018) found that experiment participants who act as homeowners may make decisions that are earnings-irrational because of the positional-good aspect of housing. This paper creates a model that seeks to minimize the positional-good aspect. As such, participants are developers who are trying to optimize by building up or out. There is a tradeoff in trying to get participants focused on this up-or-out decision; specifically, the voting treatments poorly reflect the "homevoter" formulation of the political economy of land use offered by Fischel (2004). Another economic experiment published in this journal uses the developer-participant formulation (Cypher et al., 2018).
} 


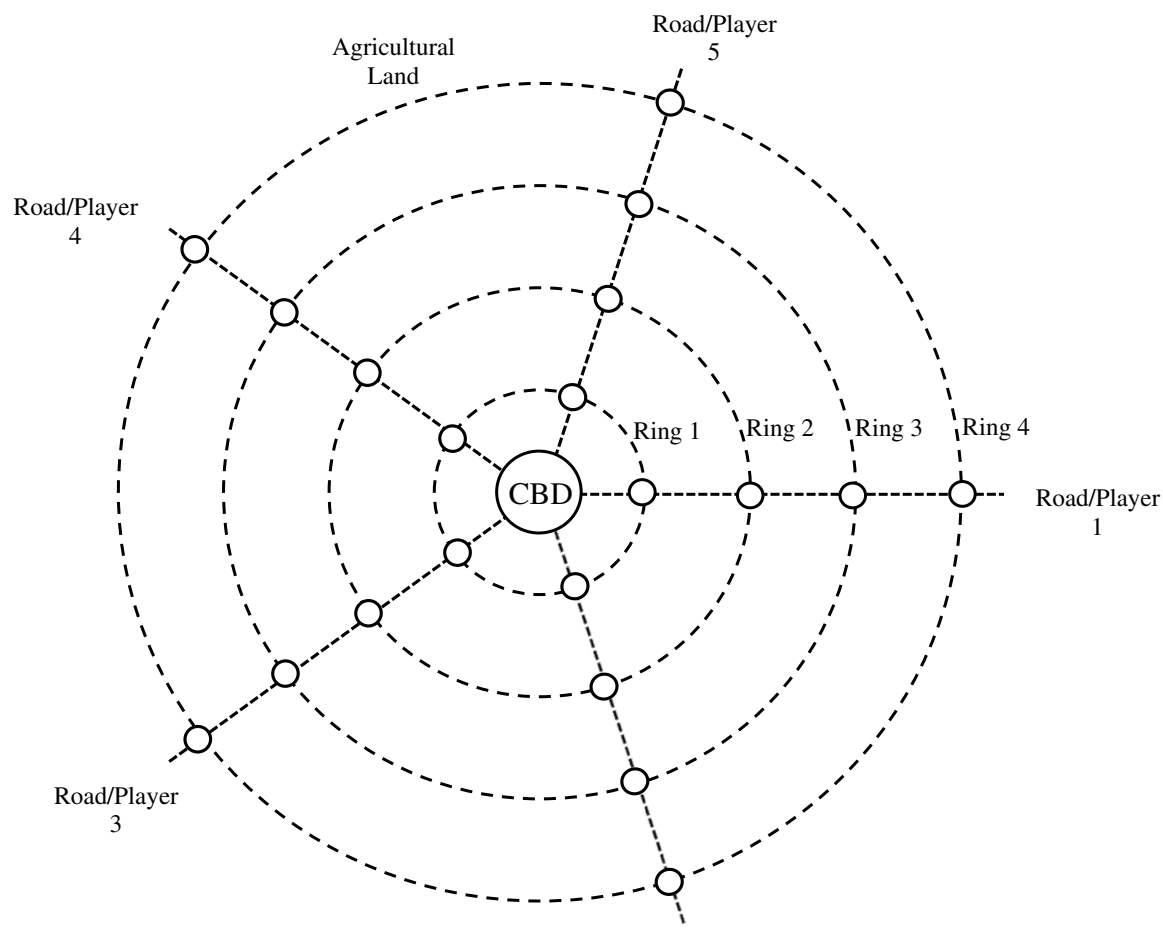

Road/Player

2

Fig. 1 Stylized city with concentric growth rings

at location $i$ for participant $j$ at period $t$. The stylized concept of placing a brick was described in terms of a Lego $@$ block as a salient symbol of land improvement. By granting one brick per period, the researchers overcame the need to have the experiment participants understand a separate production function, input costs, and complex constraints.

\section{Heterogeneous Types: Revenue and Costs}

The choice problem involves revenue, operating and maintenance costs, and taxes. All participants derived homogeneous revenue at location $i$ from each brick placement, or $R_{i j t}=200 B_{i j t}{ }^{1.6} / i$ expressed in experimental dollars. All else equal, bricks generate more revenue when bricks are closer to the CBD. Also, marginal revenue increases with each additional brick at a given location. The operating and maintenance $(\mathrm{O} \& \mathrm{M})$ costs arising from brick placement accrue in the period of placement and in each successive period: $C_{i j t}=\left(a_{1} B_{i j t}+a_{2} B_{i j t}{ }^{2}\right) / i$. The parameters $a_{1}$ and $a_{2}$ are the sole source of induced heterogeneity in the experiment, with five types (see Table 1). For all parameters, costs increase at an increasing rate with bricks at 


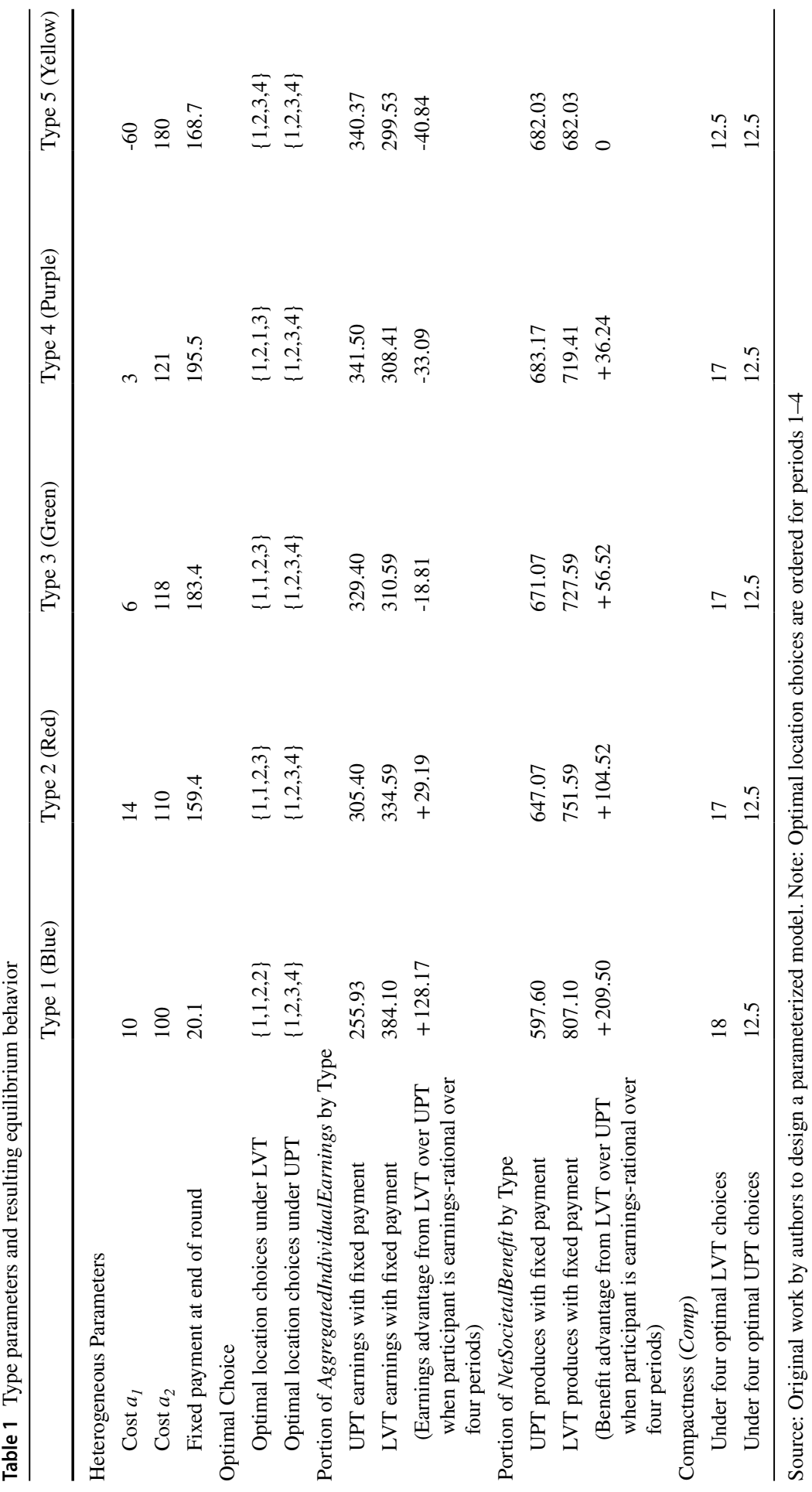


any one location. Property taxes (introduced below) change participants' location choices and earnings.

The parameterization of five types reflects modeling decisions by the researchers, who sought predicted treatment effects on efficiency, spatial development patterns, and voting behavior. However, the parameters hold several connections to real-world landowners. First, because of revenue homogeneity, a given building will earn the same market price regardless of which type builds. Also, given that each type gets one brick each period, then the types have the same endowment and production technology associated with building; one could think of very similar "box" buildings where it is easy to scale them to have small or large footprints and short or tall height. The formulation of production as a "brick" further simplifies the output of land use to be a homogeneous production unit. Second, heterogeneous costs mean some types have a comparative advantage in O\&M; these are ordered by this skill from best (Type1) to worst (Type5). Type1 has the best human capital or technology for O\&M such that they can service any building at any given location at a lower cost than the other types. These simplifications try to capture real-world settings in which relatively similar, scalable commercial or residential buildings—such as office buildings, apartment buildings, warehouses, etc.-could be placed at various distances from the CBD. More "bricks" at any location could be thought of as more intensity or vertical development.

Type1 is labeled a "LVT lover" because this type has the best O\&M technology for higher buildings and therefore will be more profitable from more intensity at any location relative to the other types. They "love" LVT in that this tax does not penalize the intensive building pattern they prefer. In contrast, Type 4 is a "UPT lover" because their technology is not skilled like Typel at O\&M. Type4 profits more when land and improvements are taxed at the same rate because they tend to have lower relative costs when there is little intensity at a location. The real-world landowner of Type 4 would have lower-than-average improvements, such as a one-story office park, while Typel would have higher intensity. Type 2 and Type 3 are the intermediate cases. Type 2 is a marginal "LVT lover" who benefits slightly from LVT, while Type3 is a marginal "UPT lover" who benefits slightly from UPT. The behavior of these two marginal types will be important in the analysis because they have the lowest opportunity cost for an earnings-irrational tax institution. Type 5 is interesting because its O\&M technology is so poor that it is indifferent between LVT and UPT in terms of location choices, though it earns more under UPT. This type may relate to low-intensity commercial land uses that have large parking lots. Their O\&M costs increase too rapidly if they add any density, so their profitability comes from having minimal possible improvements. They have the largest earnings preference for UPT because the tax rate on land is lower than LVT and they, in effect, can free ride on the public goods funded through the tax paid by their neighboring owners who have more improvements. Although the Type1 "LVT lovers" make the most efficient use of land, their comparative advantage in O\&M of bricks is unique and cannot simply be replicated by the other types. So, the social planner's problem is to devise a system of tax incentives that maximizes the net social product of the five types in the city. As explained below, the distribution of types varies so that some sessions have only one Type1, while others have two or three. 


\section{Property Values and Land Value Capitalization}

Following Banzhaf and Lavery (2010) and Duke and Gao (2018), this paper models the total property value (PV) as the sum of the land value (LV) and improvement value (IV), i.e., $\mathrm{PV}=\mathrm{LV}+\mathrm{IV}$. For simplicity, IV is modeled as the revenue from bricks at each location. Land values on one road evolve independently ${ }^{2}$ of decisions on other roads. Land value at any location $i$ is only affected by $j$ 's own choices:

$$
L V_{i j t}=\left(20 B_{i j t}+10 B_{i-1, j t}+5 B_{i+1, j t}\right) / i
$$

In this simple formulation, there are land-value spillovers, but they are internalized; this approach establishes baseline results for future studies in which the spillovers are not internalized. Land values are only positive if: (1) there is a brick at a location; and/or (2) there is a brick at an "inwards" location $i-1$ or an "outwards" location $i+1$. The CBD always has six bricks, so location 1 will always have a positive land value. The agricultural area has zero bricks. There is no negative capitalization externality in this model because $j$ 's own choices of brick location determine land value and, thus, taxes. Although the CBD effect on location $i=1$ is external to participant $j$, it (1) is not an externality that arises from participant $-j$ 's choices; (2) affects all participants in the same way; and (3) simply puts a baseline, nonzero land value on location 1. Until an endogenous voting process is introduced, the optimal choices are known with certainty. ${ }^{3}$

\section{Tax Institutions}

Two tax treatments are used. LVT assesses each location on land value alone: $\operatorname{Tax}_{i j t}{ }^{L V T}=\tau_{L V T} L V_{i j t}$. UPT taxes at the same rate for land value and for improvements, which are simplified as the revenue from bricks at each location: $\operatorname{Tax}_{i j t}{ }^{U P T}=\tau_{U P T}\left(L V_{i j t}+R_{i j t}\right)$. The tax rates were set as $\tau_{L V T}=0.90$ and $\tau_{U P T}=0.20$. The model predicts LVT generates more revenue because it is parameterized to incentivize denser, higher-value building patterns. All references to the tax plans in the experiment used neutral language whereby UPT was "Tax Plan 1" and LVT was "Tax Plan 2." Table 1 shows the set of five types with heterogeneity only in the cost functions and a fixed payment. Paid at the end of a round, the fixed payment

\footnotetext{
${ }^{2}$ A modeling challenge is to manage the degree of interaction among participants. Obviously, voting allows the participants to interact. However, based on Duke and Gao (2018), the researchers were concerned that land capitalization interdependency and tax redistribution could overpower the political economy results. As such, this paper reports an experiment with independent capitalization and no tax redistribution; tax revenue is compared by treatment but not constrained to be revenue-neutral.

${ }^{3}$ One might object that there is no salvage value in this model, which would allow one to capture appreciated land value. This modeling simplification is, in part, due to the practical need to make the decision problem as simple as possible for experiment participants. However, this simplification also can be justified in that land and bricks are supplied to the participants freely. Conceptually, the decision setting could be a manager, who takes over a going concern for four decision periods and where all management incentives reduce to the aforementioned revenue, cost, and tax processes. These incentives are net of capital cost.
} 
balanced the expected earnings among the types when earnings-rational behavior is pursued. Although the instructions explained that participants would receive the fixed payments, the payment levels ought not affect behavior because they do not affect marginal earnings from decisions. The five types were labeled by color within the experiment so that the participants would not infer any ordering.

\section{Objective Function}

Participant $j$ 's problem is to decide the location $i$ for one brick in each of four periods $t$ in a round; each round restarts the decision problem. Let the choice variable $L_{j t}$ $\epsilon\{1,2,3,4\}$ be an indicator of which one of the four locations was selected. $L_{j t}$ determines the increment of bricks on location $i$ as $X_{i j t}$ such that $X_{i j t}=1$ if $i=L_{j t}$ and 0 otherwise. The optimization problem for each participant $j$ is to maximize earnings, which is a summation of revenue, cost, and tax: $\max _{L_{j t}} \sum_{t=1}^{4} \sum_{i=1}^{4}\left[R_{i j t}-C_{i j t}-\right.$ Tax $\left._{i j t}\right]$, subject to the constraint of a single brick available in each period. When parameterized, this problem is:

$$
\begin{gathered}
\max _{L_{j t}} \sum_{t=1}^{4} \sum_{i=1}^{4}\left[200 B_{i j t}{ }^{1.6} / i-\left(a_{1} B_{i j t}+a_{2} B_{i j t}{ }^{2}\right) / i-\operatorname{Tax}_{i j t}\right] \\
\text { s.t. } B_{i j t}=B_{i j t-1}+X_{i j t} \\
X_{i j t}=1 \text { if } i=L_{j t} \text { where } L_{j t} \in\{1,2,3,4\} \text { in any } t \\
B_{i j 0=0 .}
\end{gathered}
$$

The first constraint shows how bricks increase based on those placed in the previous period. The second constraint indicates that there is only one brick available in a period and that it will be added to the location of the participants' choice. The final constraint indicates that each round starts with no bricks at any location.

Although this decision problem is relatively easy to describe to participants, it is too complex to solve by hand in the lab. There are $4^{4}=256$ possible brick placement choices in the four-period round. As such, the research team provided participants with a printout of the earnings from each of 256 possible choices; a different printout was provided for each type. This table had the top five earning decisions for LVT and for UPT highlighted, so participants could quickly identify high-earning choices.

\section{Predicted Behavior and Measures of Efficiency and Compactness}

The marginal earnings derived by a participant in a round are indicated by the variable, IndividualEarnings $s_{j}=\sum_{t=1}^{4} \sum_{i=1}^{4}\left[200 B_{i j t}{ }^{1} 6 / i-\left(a_{1} B_{i j t}+a_{2} B_{i j t}{ }^{2}\right) / i-\operatorname{Tax}_{i j t}\right]$. A related variable, AggregatedIndividualEarnings $=\sum_{j=1}^{5}$ IndividualEarnings $s_{j}$, adds up the city earnings over one round. The earnings variables measure private efficiency for the participants, capturing all wealth other than the tax revenue created by decisions in a city in a round. The social efficiency measure captures all wealth created by the participants choices, i.e., all five participants' AggregatedIndividualEarnings plus the tax revenue: NetSocietalBenefit $=\sum_{j=1}^{5} \sum_{t=1}^{4} \sum_{i=1}^{4}\left[200 B_{i j t}{ }^{1.6} / i-\left(a_{1} B_{i j t}+a_{2} B_{i j t}{ }^{2}\right) / i\right]$. 
Four location choices lead to a spatial distribution of building patterns. Many indices of sprawl exist, but this research developed a simple measure based on the metric used in gravity models of trade. City compactness measures the spatial density in terms of distance from the existing CBD:

$\operatorname{Comp}_{j}=6^{*}\left(B_{1 j t} / 1+B_{2 j t} / 2+B_{3 j t} / 3+B_{4 j t} / 4\right)$, evaluated at $t=4$.

On any road, Comp ranges from 24 , when all bricks are in location 1 , to 6 when all bricks are in location 4 . The higher the value of Comp, the more compact is the participant's "road." The five-person "city" also can be represented by a simple average of the individual road compactness values: CityComp $=\sum_{j=1}^{5} \operatorname{Comp}_{j} / 5$. Although the model is stylistic, it captures some of the main forces from the existing literature on intensification and sprawl, it develops predictions that correspond to prior findings, and the decision problem is presented in a manner that is understandable to participants.

Earnings-rational or privately optimal behavior is an ordered set of location decisions for the four periods: $\left\{L_{j 1}, L_{j 2}, L_{j 3}, L_{j 4}\right\}$ for any participant $j$-all of which can be expressed as four location decisions $i$ for simplicity. As Table 1 shows, Type 1 ought to have the most compact $(C o m p=18)$ investment under LVT, i.e., $\{1,1,2,2\}$, followed by types $2-4$, i.e., $\{1,1,2,3\}$ or $\{1,1,3,2\}, \operatorname{Comp}=17$. All types ought to pursue the same building pattern under UPT, i.e., $\{1,2,3,4\}$ with $\operatorname{Comp}=12.5$. Type 5 has no spatial treatment effect in that the optimal choice for LVT and UPT is $\{1,2,3,4\}$. IndividualEarnings $s_{j}$ vary considerably more than Comp $_{j}$ because of the type parameters. As shown in Table 1, Type1 and Type 2 earn more from their optimal choices when taxed with LVT, while the other three types prefer UPT. Table 1 presents the induced earnings advantage under LVT for each type.

\section{Varying Heterogeneity and Social Optimality}

The researchers examine how the heterogeneity of types affects the earnings, compactness, and voting outcomes from the tax institutions. TypeDistA had one of each type (1-5). This distribution predicted a 2:3 ratio of participants who "win" from LVT, so UPT should win in an earnings-rational vote. Nevertheless, AggregatedIndividualEarnings and CityComp are higher in LVT. So, LVT is potentially Pareto efficient among the five participants, but will not be selected by earnings-rational voting. This $2: 3$ contrast between what is individually and socially optimal replicates Duke and Gao's (2018) experiment, which sought to capture the principal U.S. experience with LVT. Specifically, LVT is thought to make society wealthier but tends to be voted down by communities. The current model extends prior work, in part, with two new voting distributions (3:2 and 4:1). TypeDistB had two Type1 participants and no Type5, so earnings-rational voting is 3:2 for LVT. TypeDistC had three Type1 participants and no Type4 or Type5, so earnings-rational voting is 4:1 for LVT. Type distributions did not vary within sessions to avoid participant confusion. Despite these varying predicted voting patterns, all three distributions led to a predicted outcome where LVT was best for the participants as a group in terms of aggregated cash earnings $(+64.63,+233.63$, and +394.89 , respectively). 
When one both considers participant earnings and the tax revenue, NetSocietalBenefit, the benefit advantage of LVT is even greater than it is in terms of AggregatedIndividualEarnings. As Table 1 shows, for Type1, Type2, Type3, and Type4, NetSocietalBenefit under LVT is greater than under UPT when participants make privately efficient decisions in response to the tax institution even when the institution is not the preferred institution of Type3 and Type4. The NetSocietalBenefit for Type5 is the same under LVT and UPT. Thus, NetSocietalBenefit will be positive and increasingly large as the experiment shifts from TypeDistA to TypeDistB to TypeDistC. In sum, earningsrational participant voting and brick-placement choices will lead to social efficiency in TypeDistB and TypeDistC but not TypeDistA and regardless of type distributions LVT leads to higher NetSocietalBenefit. Although AggregatedIndividualEarnings is an index of the value of production to participants, the choices also generate tax revenue that is not returned to the participants and thus NetSocietalBenefit is a better metric of social efficiency. This claim relies on an assumption about how tax revenue might be used to generate public goods: The value of the supplied public goods minus the government costs of collecting, redistributing, and spending tax revenue is greater than the tax revenue collected. Otherwise, resources are destroyed through taxation and the social efficiency calculation becomes more complicated because the amount of tax collected can affect our efficiency conclusions.

\section{Voting Treatments}

The experiment makes the tax institution endogenous in two of four rounds by allowing participants to select their preferred tax institution by a majority vote. A voting round begins with either LVT or UPT. Participants vote in the first period whether to switch to the other tax institution before they make their location choice. If a majority of 3,4 , or 5 voted to switch, then the present period and the remaining periods of the round will have the new tax institution. If 0,1 , or 2 voted to switch, then one more period of the initial tax is played and another vote is conducted in the following period. The starting tax institution was alternated for each round. Three variables derive from the voting treatments. First, Vote is an indicator of whether the round was played with a voting treatment; when Vote $=0$ the tax is exogenous. Only rounds 1 and 2 of any session have $V o t e=0$; this decision was made to avoid participant confusion with the treatments, but it will be collinear with standard learning measures (period number) in regressions because votes never occur in period 1 or 2 . Second, LVTStart indicates whether the initial period started in LVT. In the session's data, two rounds will start in LVT and two in UPT. This variable enables statistical tests of anchoring. Third, LVTPeriods $\in$ $\{0,1,2,3,4\}$ measures the number of periods in a round under LVT. In voting rounds the tax institution can switch, so one must track how much of the round was under LVT. 


\section{Data and Hypotheses}

The experiment devised an ordering of the treatments over nine sessions, which would minimize confusion. Each session had four treatment rounds reported herein. ${ }^{4}$ Each session consisted of two cities run simultaneously with the same treatments. Participants played, first, one LVT and one UPT round in nonvoting treatments and, second, one LVT and one UPT round in voting treatments for a $2 \times 2$ design. However, the UPT and LVT orders were randomized. Table A1 lists the full experiment design and the amount of data collected in each session.

\section{Experiment Sessions and Data Structure}

Data were collected at the University of Delaware's Center for Experimental and Applied Economics. The z-Tree software was used (Fischbacher, 2007) with 10 tablet computers linked to an administrator computer. Student participants were largely undergraduate business and economics majors, though others were recruited when sessions did not fill. The University of Delaware Institutional Review Board approved the protocol. Participants completed informed consent, read paper instructions, watched an instructional presentation, asked questions, and then were trained in two unpaid practice rounds. The practice rounds helped participants learn how the interface works and the basic aspects of the experiment. The first practice round had no taxation, so participants simply placed four bricks. The second practice round then introduced taxation with a different set of tax rates.

Participants completed eight rounds of four periods each—but only the first four rounds without information treatments are reported herein. ${ }^{5}$ New instructions were distributed with each treatment. Only one round was randomly selected for payment in order to magnify the incentives from each decision. A session took $1.5-2.0 \mathrm{~h}$ to complete. Table A2 presents the average participant earnings by type. The exchange rate for experimental dollars was 17: $\$ 1$. To prevent bankruptcy in the experiment, the administrator gave a participation incentive of $\$ 5$ to all who completed the experiment. A post-experiment survey (see appendix) showed that respondents tended to make earnings-rational decisions and focus on the designed aspects of the experiment (see Sauter, et al., 2016 for an experiment-survey of participants' views when the experiment aligns with their profession).

\footnotetext{
${ }^{4}$ Four subsequent information treatment rounds will be presented in another paper at the suggestion of a referee.

${ }^{5}$ Participants saw the following information during a period in the experiment. The first screen showed private information for the participant describing the status quo building and the costs and revenue of each potential brick placement in the current period. The participants made a choice on this screen. The second screen showed the results of the participant's choice and also the building pattern of four other participants. The building pattern was simply a table of five rows (for each participant) and four columns (for their locations). Each cell in this table had a number of bricks placed. Third, in voting treatments, there were two preliminary screens first asking for the individual vote and second seeing the results of the group vote.
} 
Table 2 Experiment data on tax revenue, private earnings, societal benefits, and compactness

\begin{tabular}{|c|c|c|c|c|c|c|c|c|}
\hline & & \multicolumn{2}{|c|}{ Predicted } & \multicolumn{5}{|c|}{ Observed by LVTPeriods } \\
\hline & & UPT & LVT & $\begin{array}{l}0 \\
\text { UPT } \\
\text { (t-stat) }\end{array}$ & 1 & 2 & 3 & $\begin{array}{l}4 \\
\text { LVT } \\
\text { (t-stat) }\end{array}$ \\
\hline \multirow[t]{3}{*}{ City Tax Revenue } & TypeDistA & 1708.4 & 2050.5 & $\begin{array}{l}1758.1 * * \\
N=16\end{array}$ & $\begin{array}{l}1993.6 \\
N=1\end{array}$ & - & - & $\begin{array}{l}2043.2 \\
N=7\end{array}$ \\
\hline & TypeDistB & 1708.4 & 2091.0 & $\begin{array}{l}1760.8 \\
N=10\end{array}$ & - & - & - & $\begin{array}{l}2076.0 * \\
\mathrm{~N}=14\end{array}$ \\
\hline & TypeDistC & 1708.4 & 2103.1 & $\begin{array}{l}1760.5 \\
N=6\end{array}$ & - & - & - & $\begin{array}{l}2093.0 \\
N=18\end{array}$ \\
\hline \multirow[t]{3}{*}{$\begin{array}{l}\text { Aggregated Indi- } \\
\text { vidual Earnings }\end{array}$} & TypeDistA & 845.5 & 910.2 & $\begin{array}{l}821.1^{*} \\
\mathrm{~N}=16\end{array}$ & $\begin{array}{l}633.1 \\
N=1\end{array}$ & - & - & $\begin{array}{l}862.9 \\
N=7\end{array}$ \\
\hline & TypeDistB & 909.6 & 1143.3 & $\begin{array}{l}880.3^{*} \\
\mathrm{~N}=10\end{array}$ & - & - & - & $\begin{array}{l}1123.0^{* * * *} \\
\mathrm{~N}=14\end{array}$ \\
\hline & TypeDistC & 999.4 & 1394.3 & $\begin{array}{l}954.2 \\
N=6\end{array}$ & - & - & - & $\begin{array}{l}1338.6^{* * *} \\
\mathrm{~N}=18\end{array}$ \\
\hline \multirow[t]{3}{*}{ NetSocietalBenefit } & TypeDistA & 2553.9 & 2960.7 & $\begin{array}{l}2579.2 * \\
\mathrm{~N}=16\end{array}$ & $\begin{array}{l}2626.7 \\
N=1\end{array}$ & & & $\begin{array}{l}2906.1 \\
N=7\end{array}$ \\
\hline & TypeDistB & 2618.0 & 3234.3 & $\begin{array}{l}2641.1 \\
N=10\end{array}$ & & & & $\begin{array}{l}3199.0^{* * *} \\
\mathrm{~N}=14\end{array}$ \\
\hline & TypeDistC & 2707.8 & 3497.4 & $\begin{array}{l}2714.7 \\
N=6\end{array}$ & & & & $\begin{array}{l}3431.6^{* * *} \\
\mathrm{~N}=18\end{array}$ \\
\hline \multirow[t]{4}{*}{ City Comp } & TypeDistA & 13.5 & 16.5 & $\begin{array}{l}12.8 * * * \\
\mathrm{~N}=16\end{array}$ & $\begin{array}{l}13.1 \\
\mathrm{~N}=1\end{array}$ & - & - & $\begin{array}{l}16.1^{* *} \\
\mathrm{~N}=7\end{array}$ \\
\hline & TypeDistB & 13.5 & 17.4 & $\begin{array}{l}12.9 * * \\
\mathrm{~N}=10\end{array}$ & - & - & - & $\begin{array}{l}17.2^{*} \\
\mathrm{~N}=14\end{array}$ \\
\hline & TypeDistC & 13.5 & 17.6 & $\begin{array}{l}12.8 * * * \\
\mathrm{~N}=6\end{array}$ & - & - & - & $\begin{array}{l}17.5 \\
N=18\end{array}$ \\
\hline & & & Rounds & 32 & 1 & 0 & 0 & 39 \\
\hline
\end{tabular}

Source: Original data collection by authors in nine experiment sessions. Notes: All data at the city level for five participants. Stars reflect t-statistics testing for differences between predicted values and values observed in UPT sessions (LVTPeriods $=0$ ) and LVT sessions (LVTPeriods $=4$ ); *** $\mathrm{p}<0.01$, ** $\mathrm{p}<0.05, * \mathrm{p}<0.1$. The experiment data also include the number of rounds $(\mathrm{N})$ in each realization of LVTPeriods. CityComp $=$ the average of compactness of five roads in the city

\section{Hypotheses on Earnings/Efficiency and Compactness}

Model predictions motivate hypotheses that LVT generates more participant earnings, more tax revenue, more NetSocietalBenefit, and a more compact city than UPT (Table 2). Wealth creation is measured with tax revenue, AggregatedIndividualEarnings, and NetSocietalBenefit, but we devote most of our analysis to AggregatedIndividualEarnings as our main efficiency performance metric; this is because the participants made decisions to earn money and thus maximize private efficiency rather than NetSocietalBenefit. A linear regression of AggregatedIndividualEarnings with 72 rounds as the units of analysis can be largely explained by controls on the induced values; TypeDistA and TypeDistB ought to have negative coefficients 
because they earn less in expectation than the regression's reserve category TypeDistC. Round is an integer between 1 and 4 indicating the round number in the session where AggregatedIndividualEarnings was generated, and this effect can be also examined with indicators on round numbers 1 to 4 . This controls for experience with the setting. The tax treatments require more complex controls because, in the voting treatments, the tax institution in periods 1-4 of any round can switch. LVTStart checks for anchoring and should not affect earnings. LVTPeriods should increase with earnings because LVT generates higher earnings in the parameterized model.

Another treatment variable is Vote, which indicates whether the round had a voting option. As mentioned, round measures and Vote are collinear and thus difficult to separate in the discussion. Voting should lead to higher AggregatedIndividualEarnings in TypeDistB and TypeDistC because participants can select the preferred tax. TypeDistB and TypeDistC ought to prefer LVT (3:2 and 4:1), which makes the earnings higher. TypeDistA prefers UPT, but will vote for LVT if one voter goes against earnings rationality. Alternatively, it could be that participants in all type distributions fail to select LVT even when it tends to lead to higher AggregatedIndividualEarnings, because of the aforementioned political objections to LVT. For example, some might reject LVT in this experiment because the tax rate is very high-in this case it is 90 percent versus only 20 percent for UPT. The same variables to explain the AggregatedIndividualEarnings regression can be used to explain CityComp. The logic associated with the hypotheses is similar because CityComp like AggregatedIndividualEarnings was parameterized to increase with LVT for all type distributions.

\section{Results}

Table 2 shows the experiment data on rounds played under selected institutions, with the first panel showing tax revenue for the three type distributions. Column LVTPeriods $=0$ shows the average tax revenue in rounds conducted entirely under UPT, while LVTPeriods $=4$ shows the average tax revenue in rounds conducted entirely under LVT. One-half of the rounds were conducted with endogenous tax institutions. If majority voting was allowed and all participants voted with earningsrationality, then all rounds in TypeDistA would be in the UPT cell and all rounds in TypeDistB and TypeDistC would be in the LVT cells. However, institutions were assigned exogenously in rounds 1 and 2 and could not be changed. So, 6 of 7 observed LVT rounds for TypeDistA were mandated for LVT; similarly, 6 of 10 UPT rounds for TypeDistB and 6 of 6 UPT rounds for TypeDistC were mandated for UPT - all against the types' earnings-rational tax. The remaining 1 LVT round for TypeDistA, 4 UPT rounds for TypeDistB, and 0 UPT rounds for TypeDistC under earnings-irrational taxes are endogenously selected, i.e., they reflect voting against at least one participant's earnings-rationality. In addition, the TypeDistA participants played one period of LVT (LVTPeriods $=1)$. In total, 6 of 72 rounds ( 8.3 percent) played more than one period in an endogenous tax institution against predicted voting; this is 16.7 percent of the endogenous-institution rounds. The deviations could 
reflect one or more behavioral drivers, such as: (1) errors in earnings-rational choice and (2) political/ethical objections.

Another aspect of earnings-irrational voting is the difference with which it is observed across type distributions. TypeDistC did not deviate when institutions were endogenous. However, deviations happened 16.7 percent of the time for TypeDistA and 33.3 percent for TypeDistB. A reason for this is the number of Typel participants in each type distribution. Type 1 strongly benefits from LVT and had the largest incentives. TypeDistC has three Typel participants who can use their majority to force the selection of LVT. In contrast, TypeDistA and TypeDistB have no majority with an earnings advantage of that magnitude. Type 2 through Type 5 have LVT earnings advantages from -40.83 to +29.19 . Type 3 is especially close to indifference with an LVT earnings advantages of -18.81 . Thus, one expects more uncertainty in the decision making (lower cost of voting against earnings-rationality) with TypeDistA and TypeDistB.

\section{Earnings/Efficiency Results}

First, LVT consistently generated more tax revenue, which aligns with model predictions. Table 2 presents the predictions and experimental results on average tax revenue collected. In 42 of 72 periods, average tax revenue was statistically indistinguishable from the prediction. In 16 periods, TypeDistA averaged slightly more revenue than predicted under UPT, while 14 periods under LVT in TypeDistB averaged slightly less revenue than predicted. The instances where statistical differences were not found were likely affected by a small $\mathrm{N}$, or number of rounds in which the earnings-irrational tax was endogenously selected or exogenously imposed. The point estimates suggest that UPT tends to generate more revenue than predicted. Conversely, in LVT rounds there is less tax revenue than predicted. That said, the LVT differences are not substantively large. The most important result is that LVT consistently generated far more tax revenue than UPT, as predicted. The predicted UPT tax is 1,708 while LVT averages 2,082, so the predicted tax-revenue advantage for LVT is 22 percent. Although the experiment advantage was slightly less (UPT weighted average 1,759 and LVT averages 2,078), the absolute advantage for LVT of 18 percent (or 319 units) is large.

Second, AggregatedIndividualEarnings also are higher under LVT. The second panel of Table 2 compares the predicted AggregatedIndividualEarnings to the experiment results. Rounds under LVT tended to have higher average AggregatedIndividualEarnings than UPT. Next, Table 2 shows two treatments where AggregatedIndividualEarnings were statistically indistinguishable from predictions. In 7 of 24 rounds of TypeDistA the participants played all four rounds in LVT, earning an average of 863 relative to 910 as predicted. Although this point estimate suggests the earnings were lower, there was a low $\mathrm{N}$ and no statistical difference was found. The other statistically efficient treatment was TypeDistC for UPT was also a low-N treatment. Among the remaining four treatment combinations of UPT and LVT, the resulting average earnings were statistically lower than the prediction. In sum, for TypeDistA, the average round earnings were lower than expected under UPT but not 
LVT. In TypeDistB, the average round earnings were lower in both UPT and LVT. In TypeDist $C$, the average round earnings were lower than expected under LVT but not UPT. The key points therefore are that observed earnings tended to be lower than predicted and that there is no evidence that participant earnings were less in LVT versus UPT treatments.

The collective efficiency results show that under the modeled conditions LVT is more efficient than UPT. The statistical results for NetSocietalBenefit-i.e., once the tax revenue is included-are slightly different for AggregatedIndividualEarnings (third panel of Table 2). For 16 of 24 rounds that TypeDistA played entirely in UPT, NetSocietalBenefit produced higher than expected wealth; the slightly higher tax revenue in these rounds more than compensated for the lower participant earnings. Under LVT and the TypeDistA distribution, however, average NetSocietalBenefit was indistinguishable from the overall predicted benefit, but the average NetSocietalBenefit was still $12.7 \%$ higher in LVT than UPT. NetSocietalBenefit under TypeDistB and TypeDistC was different than under TypeDistA. Specifically, UPT rounds resulted in an average NetSocietalBenefit that was statistically the same as prediction, while LVT rounds had average NetSocietalBenefit that was statistically less than prediction. The reason for this is that both the average tax revenue and the average participant earnings under LVT tended to be lower than predicted. Under UPT, there was little statistical difference from prediction found on these same two measures, but the point estimate averages were countervailing-i.e., tax revenue had a higher point estimate while participant earnings had a lower point estimate than predicted for UPT. Despite these deviations from predictions, average NetSocietalBenefit was substantially higher under LVT than UPT $(+21.1 \%$ and $+26.4 \%$ for TypeDistB and TypeDistC, respectively).

Third, Table 3 offers a controlled econometric test explaining the drivers of AggregatedIndividualEarnings (Models 1-2); this is a behavioral model derived from the experiment, so we focus on the earning accruing from the participant decisions. We present two models as a robustness check because of collinearity of Vote with the round measures; however, we focus explanations on model 1, where only Vote is used to capture the effect. ${ }^{6}$ All models have high explanatory power. Starting with LVT does not alter earnings, so there is no anchoring effect. LVT leads to higher earnings and thus efficiency, as predicted. LVTPeriods has a positive impact, so earnings are higher with more periods under LVT. The net effect of LVT is strongly positive; for instance, four periods of LVT would increase earnings by approximately 220 (at point estimates). The impact of the control variables, capturing heterogeneity and other non-treatment impacts, was as expected. The main effects of TypeDistA and TypeDistB were induced in the model to earn less than

\footnotetext{
${ }^{6}$ AggregatedIndividualEarnings Model 2 with continuous variable Round has collinearity between Round and Vote, which is unavoidable as explained above. With this constraint in mind, Model 2 will suffer from multicollinearity but without bias. To examine this imperfectly, we also estimated a model with no control on learning, Model 1. This model has omitted variable "Round", but the standard errors should be closer to the perfectly modeled standard errors and to show the robustness of all coefficient estimates other than on Vote.
} 
Table 3 OLS regression explaining experiment private earnings and compactness at the group level

\begin{tabular}{|c|c|c|c|c|}
\hline Variables & $\begin{array}{l}\text { AggregatedIndividu- } \\
\text { alEarnings } \\
\text { Model } 1\end{array}$ & $\begin{array}{l}\text { AggregatedIndividu- } \\
\text { alEarnings } \\
\text { Model } 2\end{array}$ & $\begin{array}{l}\text { CityComp } \\
\text { Model } 1\end{array}$ & $\begin{array}{l}\text { CityComp } \\
\text { Model } 2\end{array}$ \\
\hline LVTStart & $\begin{array}{l}-21.6 \\
(29.1)\end{array}$ & $\begin{array}{l}-18.5 \\
(29.3)\end{array}$ & $\begin{array}{l}-0.03 \\
(0.2)\end{array}$ & $\begin{array}{l}-0.02 \\
(0.2)\end{array}$ \\
\hline LVTPeriods & $\begin{array}{l}54.2 * * * \\
(8.7)\end{array}$ & $\begin{array}{l}52.9 * * * \\
(8.8)\end{array}$ & $\begin{array}{l}1.0 * * * \\
(0.1)\end{array}$ & $\begin{array}{l}1.0 * * * \\
(0.1)\end{array}$ \\
\hline Vote & $\begin{array}{l}125.5^{* * * *} \\
(39.8)\end{array}$ & $\begin{array}{l}86.6 \\
(55.8)\end{array}$ & $\begin{array}{l}0.5^{* * *} \\
(0.2)\end{array}$ & $\begin{array}{l}0.4 \\
(0.3)\end{array}$ \\
\hline TypeDistA & $\begin{array}{l}-275.6^{* * * *} \\
(35.8)\end{array}$ & $\begin{array}{l}-275.6^{* * * *} \\
(35.8)\end{array}$ & $\begin{array}{l}-0.7^{* * * *} \\
(0.2)\end{array}$ & $\begin{array}{l}-0.7 * * * \\
(0.2)\end{array}$ \\
\hline TypeDistB & $\begin{array}{l}-122.5^{* * * *} \\
(35.8)\end{array}$ & $\begin{array}{l}-122.5^{* * * *} \\
(35.8)\end{array}$ & $\begin{array}{l}-0.02 \\
(0.2)\end{array}$ & $\begin{array}{l}-0.02 \\
(0.2)\end{array}$ \\
\hline Vote*TypeDistA & $\begin{array}{l}-88.4 \\
(59.4)\end{array}$ & $\begin{array}{l}-93.1 \\
(59.6)\end{array}$ & $\begin{array}{l}-0.1 \\
(0.4)\end{array}$ & $\begin{array}{l}-0.1 \\
(0.4)\end{array}$ \\
\hline Vote*TypeDistB & $\begin{array}{l}-123.8 * * \\
(51.9)\end{array}$ & $\begin{array}{l}-125.5^{* *} \\
(51.9)\end{array}$ & $\begin{array}{l}-0.4 \\
(0.3)\end{array}$ & $\begin{array}{l}-0.4 \\
(0.3)\end{array}$ \\
\hline Round & & $\begin{array}{l}20.8 \\
(20.9)\end{array}$ & & $\begin{array}{l}0.1 \\
(0.1)\end{array}$ \\
\hline Constant & $\begin{array}{l}1027.8 * * * \\
(28.2)\end{array}$ & $\begin{array}{l}997.7^{* * * *} \\
(41.4)\end{array}$ & $\begin{array}{l}13.0 * * * \\
(0.2)\end{array}$ & $\begin{array}{l}12.9^{* * * *} \\
(0.3)\end{array}$ \\
\hline R-squared & 0.86 & 0.86 & 0.95 & 0.95 \\
\hline
\end{tabular}

Source: $N=72$. Original data collection by authors. Notes: Model cannot use fixed effects because of design treatments. TypeDistC, no voting, and a UPT-period start are the reserve categories. $* * * \mathrm{p}<0.01$, $* * \mathrm{p}<0.05, * \mathrm{p}<0.1$. Standard errors in parentheses

TypeDistC, the reserve category, and the results show this. The coefficients magnitudes are roughly similar to the induced value structure.

The coefficients on Vote suggest that voting increases earnings-probably because it allows a group to select its preferred tax. For TypeDistC, one clearly expects the option to vote would increase private earnings because this distribution has three "LVT lovers," who can control the majority outcome, and one marginal "LVT lover." Earnings are higher under LVT for all type distributions, but TypeDistC earns considerably more under LVT than the other distributions. Having this voting advantage, 4:1, allows TypeDistC to increase earnings by approximately 125 when allowed to vote. The result on TypeDistB and voting show that these combined effects tended to produce no extra earnings $\left(\mathrm{b}^{\text {Vote }}\right.$ and $\mathrm{b}^{\text {TypeDist }}{ }^{*}$ Vote roughly cancel each other out). As discussed above, TypeDistB should have preferred LVT 3:2 and should have earned more under LVT. However, this distribution was the most likely to select the earnings-irrational tax (Table 2 shows the city votes for UPT 4 of 12 times). TypeDistA tended to earn more when allowed to vote. This was surprising because TypeDistA was induced to vote for UPT, which would have lower AggregatedIndividualEarnings. Table 2 showed this group tended to vote too often for LVT: 1 time when LVTPeriods $=1$ and 1 time when LVTPeriods $=4$. This may explain why $\mathrm{b}^{\text {TypeDistA }}$ *Vote did not match the hypothesis of a negative effect on earnings. 


\section{Compactness Results}

The experiment results on spatial impacts collectively show that LVT leads to less sprawl than does UPT, matching the model prediction. The fourth panel of Table 2 compares the experiment results to predictions on the average compactness of the cities, by round. The model predicted that LVT would have cities 3-4 units more compact than the same cities under UPT; however, the experiment results either matched this prediction or exceeded it. Table 2 also shows that in all UPT treatment averages, the city averaged less compactness than predicted. Under LVT, it was less compact in two of three treatments. Together these results suggest a slightly greater tendency for UPT, relative to LVT, to cause sprawl. Comparing the observed outcomes for each type distribution in Table 2 shows that the average point-estimate difference of 3.3-4.7 in Comp from the tax treatments was roughly the same magnitude as predicted. In TypeDistC, LVT produced a possibly greater Comp advantage than expected (TypeDistC) because the average Comp under LVT was statistically indistinguishable from the prediction, while the average Comp under UPT was statistically less than the prediction. As with the efficiency results, these observed differences in behavior from the type distributions likely reflect differences in the magnitude of incentives; TypeDistC has three Type1 participants and two marginal types ( 2 and 3 ), so a majority have the clearest incentives about where to build and what tax plan to select. In contrast, TypeDistB has only two Typel participants and TypeDistA has only one Type1. Collectively, the TypeDistA and TypeDistB sessions are more likely to face uncertainty in their decision-making and, thus, one expects the resulting average compactness to be less in line with predictions than TypeDistC sessions. A more complete causal explanation of these differences is shown in the regression results that explain CityComp.

Table 3 presents two regressions that explain CityComp. The collinearity of Round and Vote is the same as in the AggregatedIndividualEarnings regressions. Thus, the following part explains CityComp Model 1. As with AggregatedIndividualEarnings, the coefficients on the type distribution controls tend to be significant, but the unexpected result is TypeDistB in the second model. A reason why the TypeDistB coefficient lacks significance may be that (as in Table 2) a large number $(\mathrm{N}=10)$ of 24 rounds were played in UPT, with 4 selected by a city against the majority interest. Table 3 shows that LVT leads to more compactness because an additional LVT period (LVTPeriods) leads to 1 point of Comp. The prediction (from Table 2) was that, on average, LVT would lead TypeDistA to 3 more CityComp points, TypeDistB to 3.9 more CityComp points, and TypeDistC to 4.1 more CityComp points. The coefficients show a similar treatment effect, where 4 LVT periods lead to roughly 3.3 Comp points for TypeDistA $\left(4 \mathrm{~b}^{\text {LVTPeriods }}+\mathrm{b}^{\text {TypeDistA }}\right)$ and about 4 for TypeDistB $\left(4 \mathrm{~b}^{\text {LVTPeriods }}+\mathrm{b}^{\text {TypeDistB }}\right)$ and TypeDistC $\left(4 \mathrm{~b}^{\text {LVTPeriods }}\right)$.

The collective behavioral results (Tables 2 and 3) on compactness suggest that participants were either making decisions that resulted in as much or less compactness than predicted; there was no evidence of behavior leading systematically and substantively to more compactness than predicted. There were several situations most likely to produce predicted compactness. First, when the land characteristics have more "LVT lover" types. These settings would be more likely to observe 
Table 4 Average votes for Land Value Taxation (LVT) by type distribution

\begin{tabular}{ll}
\hline & $\begin{array}{l}\text { Votes } \\
(\mathrm{t}-\mathrm{stat})\end{array}$ \\
\hline TypeDistA & 1.88 \\
(predicted 2) & $\mathrm{N}=34$ \\
& $(-0.70)$ \\
TypeDistB & 2.75 \\
(predicted 3) & $\mathrm{N}=36$ \\
& $(-1.60)$ \\
TypeDistC & 4.13 \\
(predicted 4) & $\mathrm{N}=30$ \\
& $(0.94)$ \\
Voting Periods & 100 \\
\hline
\end{tabular}

Note: Only round 3 and round 4 include a voting mechanism that endogenously determines the tax plan used in following periods. Each of the 2 rounds could have 1 to 4 period(s) of voting. The average group votes for LVT are calculated by type distribution. The number of periods with votes are listed underneath the average group votes $(\mathrm{N})$. There is a maximum of 4 voting periods in any round, but there may be fewer. For this reason, the number of voting periods is not the same across treatments. $* * * \mathrm{p}<0.01$, ** $\mathrm{p}<0.05$, $* \mathrm{p}<0.1$

compact building patterns, and this tendency would be higher under LVT. Second, LVT tended to produce more compactness than UPT at levels predicted or at levels slightly exceeding predictions. This provides some experimental evidence to the longstanding claim that LVT does indeed reduce sprawl, and the behavioral evidence suggests that the impact may even be larger than expected.

\section{Group Voting Behavior}

Table 4 presents the group voting data averaged by treatment and the predicted votes ( 2 votes for TypeDistA, 3 votes for TypeDistB, and 4 votes for TypeDistC). Votes for LVT were statistically indistinguishable from predictions for all three type distributions. Collectively, the voting results show that participants tend to favor LVT only when the underlying incentive structure has a majority of LVT lovers (TypeDistB and TypeDistC). In other words, LVT was preordained to be the most privately efficient institution for the participants, and yet the results suggest that groups only select LVT when the majority of participants win from LVT. There is no evidence of a universal objection to LVT.

\section{Policy Implications and Conclusion}

This induced-value experiment explored the political economy of land tax institutions, particularly how those taxes affect efficiency and sprawl when endogenously selected. The experiment results on LVT's advantage on efficiency and compactness 
closely match predictions, but the deviations also show the benefits of additional insight from behavioral economics. Although participant choices lead to slightly less efficiency and compactness than predicted, the results offer evidence that LVT leads to more efficiency and less sprawl.

The most interesting results relate to endogenous institutions. The group voting results showed that voting tended to be earnings-rational, though a substantive minority of group results deviated from earnings-rationality. This included TypeDistA, which was induced to support UPT against social efficiency. Thus, there was little evidence of an unequivocal earnings-irrational, equity-based rejection of LVT at the group level. However, the group results at the margin of UPT/LVT support (TypeDistA and TypeDistB) show some tendencies (16.7 and 33.3 percent respectively) to vote against earnings-rationality. Therefore, LVT promoters may benefit from anticipating a modest, substantive level of political objections, particularly because UPT is the status quo in most U.S. jurisdictions and therefore LVT acceptance will require a number of marginal LVT lovers to understand that they are truly better off under a new tax institution.

Obviously, this experiment is built upon a stylized model, but it does mimic some fundamental processes at work in actual land markets. The most extreme assumption in this model is that decisions made by one individual lead to independent landvalue capitalization. This assumption was pragmatic-to make the model more understandable to the participants and ensure that the researchers could solve for behavioral predictions. Other assumptions are less severe, such as the model parameterization where LVT would be more efficient and lead to less sprawl than UPT. The literature supports this approach, and it is difficult to conceptualize a model of this type where LVT would lead to less efficiency and more sprawl. Nevertheless, the magnitude of the efficiency and compactness advantages can be adjusted with the parameterization; therefore, the size of the efficiency and compactness advantages found for LVT are not the primary message. With the parameterization, the results showed that groups will be unlikely to reach the efficient frontier. Small deviations from optimal choice are going to be made either by mistake or for some non-earnings reason. As such, the optimum will not tend to be achieved, and yet the results suggest no systematic pattern whereby the deviations are larger in LVT than in UPT. Moreover, there was evidence that in some situations UPT may even have a systematic tendency to deliver more-than-expected sprawl. This result means that policy makers gain some confidence, though not certainty, that LVT will deliver on the promises of efficiency and compactness - as long as the underlying distributions of types in the real world match the setting developed here.

The parameterization, however, allows the researchers to test, first, whether the efficiency and compactness advantages would be as great or greater than predictions when using a behavioral economics setting - a setting that allowed for patterns of behavior that do not perfectly align with earnings-rationality. Second, the setting allows the researchers to explore how group heterogeneity affects endogenous institutional choice.

How does group heterogeneity relate to the real world? Consider one case: The majority of landowners in a jurisdiction tend to have preferences that match Type1 and Type2, i.e., the owners currently have a relative skill in intensively developing 
land. This might be a situation where agglomeration economies for intensive development exist. The evidence herein suggests that LVT is the most efficient tax institution. There will be large efficiency and sprawl cost to using UPT. Voting for the jurisdiction's preferred tax will tend to lead to LVT-as long as the owners understand their earnings under both taxes. One possibility for future research is to examine what would happen to voting if residents had an incomplete understanding of their future earnings under the alternate tax regime, which would typically be LVT as UPT is the status quo tax. Such research could further explore whether publicgood type efforts to provide this information helps groups see the advantage of LVT.

Another case might be one that matches TypeDistA. For instance, a city is distressed with very little remaining economic activity in the CBD and more activity sprawling into the suburban communities. This could potentially be a market signal that the region has a comparative disadvantage in intensive development. In terms of this paper's model, this situation might match TypeDistA in that LVT would create more "losers" than "winners" but the overall effect would be efficient. If LVT were proposed, there likely would be substantial opposition. Voting may not deliver LVT. A further concern, but one that goes beyond the model, is that there may be some communities where LVT is not the most efficient tax institution. If a community has a dramatic comparative disadvantage in intensive development, then LVT possibly would even fail to promote efficiency. There is probably some lesson in the pattern of status-quo density for one to infer the relative advantage/disadvantage the community would have in further promotion of intensive land use. Future experiments ought to vary the revenue function to examine heterogeneity in greater depth, which would build more robust conclusions on economic activity.

A final case may be a city that is already densely developed with vibrant economic activity. Presumably this signals the profitability of intensity, so LVT could potentially enhance this profitability further. The already-dense city could resist the urge to sprawl and develop greater density. Voting should lead to LVT.

A limitation of this study is that the experimental evidence probably is quite sensitive to assumptions about the land-market structure. If land market decisions do not capitalize independently, is a systematic tendency introduced that invalidates these results? Agricultural land ought to be largely independent of improvements in the sense that the price when agriculture is a highest-and-best use derives from the commodities produced rather than a neighbor's investment. In urban settings, there are pervasive interdependencies, such as economies of agglomeration, and land values are constantly adjusting to changes in economic activity among proximate parcels. That said, it would be incorrect to interpret this paper's results as speaking against LVT. The model was built without a negative capitalization externality, and it did not allow for a corresponding increase in the profitability of the buildings from adjacent development. Thus, the improvement values are artificially constrained to be fixed to make the experimental conditions understandable to participants.

Supplementary Information The online version contains supplementary material available at https://doi. org/10.1007/s11146-021-09875-9. 
Acknowledgements The authors are grateful for the funding from the Lincoln Institute of Land Policy and support from its fellows and staff members, including Joan Youngman, Semida Munteanu, and Mark Skidmore. Support also came from the Alabama Agricultural Experiment Station and USDA-NIFA Hatch.

Data Availability Duke, Joshua, 2021, "Replication Data for: "Land Value Taxation: A Spatially Explicit Economic Experiment with Endogenous Institutions,"” https://doi.org/10.7910/DVN/BMHTCD, Harvard Dataverse, V1, UNF:6:YrMRmkjOhtzsc98J6duNtg== [fileUNF]

Open Access This article is licensed under a Creative Commons Attribution 4.0 International License, which permits use, sharing, adaptation, distribution and reproduction in any medium or format, as long as you give appropriate credit to the original author(s) and the source, provide a link to the Creative Commons licence, and indicate if changes were made. The images or other third party material in this article are included in the article's Creative Commons licence, unless indicated otherwise in a credit line to the material. If material is not included in the article's Creative Commons licence and your intended use is not permitted by statutory regulation or exceeds the permitted use, you will need to obtain permission directly from the copyright holder. To view a copy of this licence, visit http://creativecommons.org/licen ses/by/4.0/.

\section{References}

Banzhaf, H. S., \& Lavery, N. (2010). Can the land tax help curb urban sprawl? Evidence from growth patterns in Pennsylvania. Journal of Urban Economics, 67(2), 169-179.

Bourassa, S. C. (2009). "The Political Economy of Land Value Taxation.” In Land Value Taxation: Theory, Evidence, and Practice, ed. R. F. Dye, \& R. W. England. Lincoln Institute of Land Policy.

Bowman, J. H., \& Bell, M. E. (2008). Distributional consequences of converting the property tax to a land value tax: Replication and extension of England and Zhao. National Tax Journal, 61(4), 593-607.

Brueckner, J. K., \& Kim, H.-A. (2003). Urban sprawl and the property tax. International Tax and Public Finance, 10(1), 5-23.

Chapman, J. I., Johnston, R. J., \& Tyrrell, T. J. (2009). Implications of a land value tax with error in assessed values. Land Economics, 85(4), 576-586.

Choi, K.-W., \& Sjoquist, D. L. (2015). Economic and spatial effects of land value taxation in an urban area: An urban computable general equilibrium approach. Land Economics, 91(3), 536-555.

Cypher, M., McKay Price, S., Robinson, S., \& Seiler, M. J. (2018). Price signals and uncertainty in commercial real estate transactions. The Journal of Real Estate Finance and Economics, 57, 246-263.

DiMasi, J. A. (1987). The effects of site value taxation in an urban area: A general equilibrium computational approach. National Tax Journal, 40(4), 577-590.

Duke, J. M., \& Gao, TianHang. (2018). An experimental economics investigation of the land value tax: Efficiency, acceptability, and positional goods. Land Economics, 94(4), 475-495.

England, R. W., \& Zhao, M. Q. (2005). Assessing the distributive impact of a revenue-neutral shift from a uniform property tax to a two-rate property tax with a uniform credit. National Tax Journal, 58(2), $247-260$.

England, R. W., \& Ravichandran, M. (2010). Property taxation and density of land development: A simple model with numerical simulations. Eastern Economic Journal, 36(2), 229-238.

England, R. W., Zhao, M. Q., \& Huang, J.-C. (2013). Impacts of property taxation on residential real estate development. Journal of Housing Economics, 22, 45-53.

Fischbacher, U. (2007). Z-Tree: Zurich toolbox for ready-made economics experiments. Experimental Economics, 10(2), 171-178.

Fischel, W. (2004). An economic history of zoning and a cure for its exclusionary effects. Urban Studies, $41(2), 317-340$.

Fischel, W. A. (2015). Zoning Rules! The Economics of Land Use Regulation. Lincoln Institute of Land Policy.

Gemmell, N., Grimes, A., \& Skidmore M. (2019). "Do local property taxes affect new building development? Results from a quasi-natural experiment in New Zealand." Journal of Real Estate Finance and Economics, 58, 310-333.

Plassmann, F., \& Tideman, T. N. (2000). A markov chain monte carlo analysis of the effect of two-rate property taxes on construction. Journal of Urban Economics, 47(2), 216-247. 
Plummer, E. (2009). "Fairness and Distributional Issues." In Land Value Taxation: Theory, Evidence, and Practice, ed. R. F. Dye, \& R. W. England. Lincoln Institute of Land Policy.

Plummer, E. (2010). Evidence on the distributional effects of a land value tax on residential households. National Tax Journal, 63(1), 63-92.

Pollock, R. L., \& Shoup, D. C. (1977). The effect of shifting the property tax base from improvement value to land value: An empirical estimate. Land Economics, 53(1), 67-77.

Sauter, P. A., Mußhoff, O., Möhring, B., \& Wilhelm, S. (2016). "Faustmann vs. real options theory - an experimental investigation of foresters' harvesting decisions. Journal of Forest Economics, 24, 1-20.

Song, Y., \& Zenou, Y. (2006). Property tax and urban sprawl: Theory and implications for US cities. Journal of Urban Economics, 60(3), 519-534.

Youngman, J. (2016). A Good Tax: Legal and Policy Issues for the Property Tax in the United States. Lincoln Institute of Land Policy.

Publisher's Note Springer Nature remains neutral with regard to jurisdictional claims in published maps and institutional affiliations. 